University of Wollongong

Research Online

Faculty of Engineering and Information

Faculty of Engineering and Information

Sciences - Papers: Part A

Sciences

$1-1-2008$

On generalization of constitutive models from two dimensions to three dimensions

N Khalili

University of New South Wales

M D. Liu

University of New South Wales, martindl@uow.edu.au

Follow this and additional works at: https://ro.uow.edu.au/eispapers

Part of the Engineering Commons, and the Science and Technology Studies Commons

Research Online is the open access institutional repository for the University of Wollongong. For further information contact the UOW Library: research-pubs@uow.edu.au 


\title{
On generalization of constitutive models from two dimensions to three dimensions
}

\begin{abstract}
In this paper, a study is made of the generalization of constitutive models for geomaterials from twodimensional stress and strain states to three-dimensional stress and strain states. Existing methods of model generalization are reviewed and their deficiencies are highlighted. A new method is proposed based on geometries of the model imprints on two normal planes. Using the proposed method, various three dimensional failure criterions suitable for geomaterials are implemented directly into a two dimensional model and the generalized model is identical to its original form for the axially symmetric condition. To demonstrate the application of the proposed method, the Modified Cam Clay model is extended using the Matsuoka-Nakai failure criterion. Simulations of soil behaviour for loading in the principal stress space are presented and analyzed
\end{abstract}

\section{Keywords}

three, dimensions, constitutive, two, models, generalization

\author{
Disciplines \\ Engineering | Science and Technology Studies
}

\section{Publication Details}

Khalili, N. \& Liu, M. D. (2008). On generalization of constitutive models from two dimensions to three dimensions. International Journal for Numerical and Analytical Methods in Geomechanics, 32 2045-2065. 


\title{
On Generalization of Constitutive Models from 2D to 3D
}

\author{
N. Khalili* and M. D. Liu \\ School of Civil and Environmental Engineering, \\ The University of New South Wales, \\ Sydney, NSW, 2052, Australia \\ *e-mail: n.khalili@unsw.edu.au
}

\begin{abstract}
In this paper, a study is made of the generalization of constitutive models for geomaterials from two-dimensional stress and strain states to three-dimensional stress and strain states. Existing methods of model generalization are reviewed and their deficiencies are highlighted. A new method is proposed based on geometries of the model imprints on two normal planes. Using the proposed method, various three dimensional failure criterions suitable for geomaterials are implemented directly into a two dimensional model and the generalized model is identical to its original form for the axially symmetric condition. To demonstrate the application of the proposed method, the Modified Cam Clay model is extended using the Matsuoka-Nakai failure criterion. Simulations of soil behaviour for loading in the principal stress space are presented and analyzed.
\end{abstract}

\section{KEY WORDS:}

Constitutive relations, plasticity, model generalization. 


\section{INTRODUCTION}

A constitutive model, which provides the stress and strain response of a material element in a three dimensional space, is essential for numerical analyses of real-life boundary value problems that involve force and deformation of a system. Such a model is perhaps the most important element in a computational code controlling the predictive accuracy and the relevance of the numerical solutions obtained. Both stress and strain can be described in terms of a second order Cartesian tensor. Nevertheless, a complete characterization of the relationship between the stress and strain tensors is very complicated, and is more often than not unattainable through conventional engineering testing methods. Thus, constitutive models are frequently developed for relatively simple conditions such as two dimensional cases, where experimental data are available for their formulation and validation.

To provide reliable simulations of practical boundary value problems, a two dimensional model must necessarily be extended into a general model in the three dimensions. Several approaches have been proposed in the literature. Among the notable contributions include the work of Lagioia et al (1996), Gajo and Muir Wood (1999), Liu and Carter (2002), Wheeler et al (2003), Dafalias et al (2006), among others. However, most of these approaches have been based on certain simplifying assumptions and their applicability and effectiveness have yet to be examined in detail.

In this paper, a study is made of the existing methods of model generalization. Their advantages and deficiencies are examined, and a more general approach for the extension of two dimensional models into three dimensions is proposed based on the imprints of the model on two normal planes within the three dimensional space. To demonstrate the 
workings of the method, the Modified Cam Clay model is extended with Matsuoka-Nakai criterion at the critical state as the other imprint of the three-dimensional model. Typical simulations for loading in the principal stress space are presented. Comparisons are made between the performance of the proposed method and that of the existing methods. It is shown that using the approach proposed the deficiencies of existing methods are eliminated.

Notation: Subscript $2 \mathrm{D}$ is used to indicate parameters for two dimensional stress and strain states. $\sigma_{1}^{\prime}, \sigma_{2}^{\prime}$ and $\sigma_{3}^{\prime}$ are the three principal stresses, and $\varepsilon_{1}, \varepsilon_{2}$ and $\varepsilon_{3}$ are the three coaxial principal strains. Tensor quantities are identified by boldface letters. Soil state is described by a second order stress tensor $\boldsymbol{\sigma}^{\prime}$ and a second order strain tensor $\boldsymbol{\varepsilon}$ in the Cartesian coordinate system. Symbols "." and ":" denote the inner products of two tensors with single and double contraction, respectively. $\operatorname{tr}$ is the trace operator, with $\operatorname{tr} \boldsymbol{\sigma}^{\prime}=\sigma_{i i}^{\prime}$, where summation over the repeated indices is used. $\boldsymbol{\delta}$ is the second order identity tensor. The deviatoric stress tensor $\mathbf{s}$ is given by $\mathbf{s}=\boldsymbol{\sigma}^{\prime}-\left(\frac{\operatorname{tr} \boldsymbol{\sigma}^{\prime}}{3}\right) \boldsymbol{\delta}$; and the deviatoric strain tensor $\mathbf{e}$ is given by $\mathbf{e}=\boldsymbol{\varepsilon}-\left(\frac{\operatorname{tr} \boldsymbol{\varepsilon}}{3}\right) \boldsymbol{\delta}$. The commonly adopted two dimensional stress invariants $\left(p_{2 D}^{\prime}, q_{2 D}\right)$ which correspond to the conventional triaxial conditions, i.e., $\sigma_{2}^{\prime}=\sigma_{3}^{\prime}, \varepsilon_{2}=\varepsilon_{3}$ are written as

$p_{2 D}^{\prime}=\frac{1}{3}\left(\sigma_{1}^{\prime}+2 \sigma_{3}^{\prime}\right)$

$q_{2 D}=\sigma_{1}^{\prime}-\sigma_{3}^{\prime}$, 
The corresponding work conjugate strains are volumetric (isotropic) strain $\varepsilon_{v, 2 D}$ and the shear (deviatoric) strain $\varepsilon_{d, 2 D}$ given by

$\varepsilon_{v, 2 D}=\varepsilon_{1}+2 \varepsilon_{3}$

$\varepsilon_{d, 2 D}=\frac{2\left(\varepsilon_{1}-\varepsilon_{3}\right)}{3}$

The two dimensional stress ratio $\eta_{2 D}$ is in turn defined as

$\eta_{2 D}=\frac{q_{2 D}}{p_{2 D}}=\frac{3\left(\sigma_{1}^{\prime}-\sigma_{3}^{\prime}\right)}{\left(\sigma_{1}^{\prime}+2 \sigma_{3}^{\prime}\right)}$,

In the three dimensional stress and strain space, the mean effective stress $p^{\prime}$ is written as

$p^{\prime}=\frac{1}{3}\left(\sigma_{1}^{\prime}+\sigma_{2}^{\prime}+\sigma_{3}^{\prime}\right)=\frac{t r \sigma^{\prime}}{3}$,

The definition of the general shear stress, however, is usually dependent on the criterion selected to describe the ultimate state of the material. The general shear stress corresponding to von Mises criterion is given as 


$$
\begin{aligned}
q_{V M} & =\frac{1}{\sqrt{2}} \sqrt{\left(\sigma_{1}^{\prime}-\sigma_{2}^{\prime}\right)^{2}+\left(\sigma_{2}^{\prime}-\sigma_{3}^{\prime}\right)^{2}+\left(\sigma_{3}^{\prime}-\sigma_{1}^{\prime}\right)^{2}} \\
& =\frac{\sqrt{6}}{2} \sqrt{(\mathbf{s}: \mathbf{s})}
\end{aligned}
$$

The volumetric strain increment $\varepsilon_{v}$ and the deviatoric strain increment $\varepsilon_{d}$ are defined as

$$
\varepsilon_{v}=\varepsilon_{1}+\varepsilon_{2}+\varepsilon_{3}=\operatorname{tr} \boldsymbol{\varepsilon}
$$

$$
\begin{aligned}
\varepsilon_{d}= & \frac{\sqrt{2}}{3} \sqrt{\left(\varepsilon_{11}-\varepsilon_{22}\right)^{2}+\left(\varepsilon_{22}-\varepsilon_{33}\right)^{2}+\left(\varepsilon_{33}-\varepsilon_{11}\right)^{2}} \\
= & \frac{\sqrt{6}}{3} \sqrt{(\mathbf{e}: \mathbf{e})}
\end{aligned}
$$

\section{REVIEW OF EXISTING METHODOLOGIES}

Except for a few models that have been formulated in the three dimensional space (e.g., Lade, 1977; Nakai and Mihara, 1984), most three dimensional constitutive models for geomaterials are derived from two dimensional models initially developed in the $q_{2 D} p_{2 D}^{\prime}$ plane. The methods currently employed for the model generalization can be divided into three main groups according to the way the generalized shear stress ratio is defined. Hereafter, they are referred to as: (1) von Mises method, (2) modified von Mises method, and (3) generalized shear stress ratio method. They are examined in turn in the following sub-sections. 


\subsection{Von Mises method}

In the von Mises method, the generalized shear stress is derived from the von Mises failure criterion using equation (7), and the corresponding generalized shear stress ratio $\eta_{V M}$ is defined as the ratio of the shear stress $q_{V M}$ over the general mean effective stress as follows,

$\eta_{V M}=\frac{q_{V M}}{p^{\prime}}=\frac{3 \sqrt{6}}{2}\left(\frac{\sqrt{\mathbf{s}: \mathbf{s}}}{t r \boldsymbol{\sigma}^{\prime}}\right)$.

As a result, constitutive equations and models initially derived in a two dimensional plane can simply be rewritten in terms of the generalized definitions (6) and (10). As an example, let a yield surface for a two dimensional model be described by

$f\left(p_{2 D}^{\prime}, \eta_{2 D}, M_{2 D}, p_{o}^{\prime}\right)=0$,

where $p_{2 D}^{\prime}$ and $\eta_{2 D}$ are the mean effective stress and shear stress ratio for two dimensional stress states, $M_{2 D}$ is the value of $\eta_{2 D}$ at a critical state, and $p_{o}^{\prime}$ represents the size of the yield surface.

The corresponding three dimensional yield surface will keep the mathematical format of the two dimensional surface and is obtained by substituting stress parameters $p_{2 D}^{\prime}$ and $\eta_{2 D}$ with the generalized mean effective stress $p^{\prime}$ and shear stress ratio $\eta_{V M}$. Thus, the following general surface is obtained 


$$
f\left(p^{\prime}, \eta, M_{2 D}, p_{o}^{\prime}\right)=f\left[\frac{\operatorname{tr} \boldsymbol{\sigma}^{\prime}}{3}, \frac{3 \sqrt{6}}{2}\left(\frac{\sqrt{\mathbf{s}: \mathbf{s}}}{t r \boldsymbol{\sigma}^{\prime}}\right), M_{2 D}, p_{o}^{\prime}\right]=0
$$

which represents the revolution of the two dimensional yield surface about the hydrostatic $p^{\prime}$ axis, Fig 1. The von Mises criterion is suitable for describing the mechanical properties of materials in which the failure is independent of the third invariant of the stress deviators. In fact, the failure of this type is facilitated when the rate of distortional deformation reaches a critical value (Collins and Houlsby, 1997). However, geomaterials are fundamentally frictional in nature, and their ultimate state is controlled by the mobilization of both the second and third invariants of the deviatoric stress tensor.

Strictly speaking, the von Mises method of model generalization is not appropriate for geomaterials. Even though, it has been widely used for both engineering predictions and theoretical study (e.g., Potts and Zdravkovic, 1999; Kavvadas and Amorosi, 2000; Liu and Carter, 2002, Wheeler et al, 2003; Tsutsumi and Hashiguchi, 2005). An appealing aspect of this method is its simplicity for both mathematical manipulation and physical understanding. Also, the extension of a model from two to three dimensions is obtained through a strikingly simple process, particularly for the examination of a model using the principles of thermodynamics (Collins and Houlsby, 1997).

\subsection{Modified von Mises method}

It has been widely observed that the ultimate surfaces in the deviatoric plane for geomaterials vary with the mean effective stress, and normally lie in the range defined 
by the von Mises and the Mohr-Coulomb criteria, Fig. 2 (e.g., Yamada, 1979; Callisto and Calabresi, 1998; Liu and Carter, 2003). In order to capture numerically the trend of the strength variation in the deviatoric plane, a modification of the von Mises method is typically made. Instead of being kept constant, the value of the critical state strength $M_{2 D}$ is allowed to vary with the relative magnitudes of the principal stresses. The relative magnitudes of the principal stresses are represented by the Lode's angle, $\theta$. Therefore, the critical state strength is written as

$$
M=\frac{q}{p^{\prime}}=M(\theta)
$$

Meanwhile the generalized shear stress ratio $\eta$ is retained the same as that defined for the von Mises method, equation (10). A number of equations for simulating this type of variation have been proposed (e.g., Argyris et al, 1974; Sheng et al, 2000; Arduino and Macari, 2001). The equation proposed by Argyris (1974) has been widely used and is introduced here as an example to illustrate the process of model generalization using this method (e.g., Gajo and Muir Wood; 1999; Potts, 2003; Dafalias et al 2006).

The Lode's angle is defined as

$$
\sin (3 \theta)=\frac{\sqrt{6}(\mathbf{s} \cdot \mathbf{s}): \mathbf{s}}{(\mathbf{s}: \mathbf{s})^{3 / 2}}
$$

in which $\theta$ takes a value of $\pi / 6$, for triaxial compression test, and $-\pi / 6$, for triaxial 
extension.

Argyris (1974) proposed the following equation for the critical state strength

$$
M(\theta)=\frac{2 m}{(1+m)-(1-m) \sin 3 \theta} M_{2 D},
$$

where $M_{2 D}$ is the value of the critical state strength obtained from the conventional triaxial compression test. Parameter $m$ is in turn defined as,

$$
m=\frac{M_{e x t}}{M_{2 D}}=\left(\frac{\sin \varphi_{e}}{\sin \varphi_{c}}\right)\left(\frac{3-\sin \varphi_{c}}{3+\sin \varphi_{e}}\right),
$$

where $M_{e x t}$ is the value of the critical state strength for the conventional triaxial extension test. $\varphi_{c}$ and $\varphi_{e}$ are the critical state friction angles measured in the conventional triaxial compression and extension tests, respectively.

As a result, two dimensional constitutive equations and models can be rewritten in terms of the generalized mean effective stress, given by equation (6), shear stress ratio, given by equation (10), and the variable critical state strength $M(\theta)$. Again using the yield surface given by equation (11), as an example, the following generalized yield surface is obtained

$$
f\left[p^{\prime}, \eta, M(\theta), p_{o}^{\prime}\right]=f\left[\frac{\operatorname{tr} \boldsymbol{\sigma}^{\prime}}{3}, \frac{3 \sqrt{6}}{2}\left(\frac{\sqrt{\mathbf{s}: \mathbf{s}}}{t r \boldsymbol{\sigma}^{\prime}}\right), \frac{2 m}{(1+m)-(1-m) \sin 3 \theta} M_{2 D}, p_{o}^{\prime}\right]=0
$$


The modified von Mises method with various equations for $M(\theta)$ is relatively simple and can successfully describe the variation of the stiffness as well as the strength of geomaterials with the intermediate principal stress. However, two deficiencies exists in this method.

Firstly, for an originally convex yield surface in the two dimensional stress plane, the generalized yield surface in the principal stress space may not be convex for materials with relatively high critical stress ratios. To demonstrate this, two example surfaces are shown in Fig. 3, using Argyris equation: one for $\phi_{c s}=30^{\circ}$, or $M_{2 D}=1.2$, and the other for $\phi_{c s}=40^{\circ}$, or $M_{2 D}=2$. Critical state friction angles for triaxial compression and extension tests are assumed to be equal in both cases, although this does not affect the point being made here. It can be seen that serious concavity occurs for the yield surface in the principal stress space for both friction angles. Although some criteria have been proposed to treat the problem of concavity with some success (e.g., Sheng et al, 2000; Arduino and Macari, 2001), to the authors' knowledge currently there are no equations that completely remove this deficiency from the modified von Mises method.

The second one is subtle. As mentioned previously, the von Mises criterion is suitable for describing the mechanical properties of materials in which the failure is independent of the third invariant of the stress deviators. It can be seen that in the criterion the shear stress ratio on the octahedral plane controls the shearing deformation of a material as well as its strength. Then, the following situation may occur. For some stress states, the shear stress ratio on the octahedral plane may be rational, i.e., the mean stress is positive, and valid, i.e., below the strength of the material. However, a corresponding normal stress may be 
become negative. The existence of such a stress state in frictional geomaterials is irrational.

\subsection{Generalized shear stress ratio method}

The rational behind the generalized shear stress ratio method is straightforward. As noted previously, the stress parameters adopted in almost all two dimensional constitutive models for geomaterials can be reduced into two components: 1) the mean effective stress, denoted as $p^{\prime}{ }_{2 \mathrm{D}}$, describing the effect of stress level, and 2) the shear stress ratio, denoted as $\eta_{2 D}$, describing the effect of shear stress ratio level, as given in equation (5). It would therefore be logical and also attractive if a generalized mean effective stress parameter $p^{\prime}$ and a generalized shear stress ratio parameter $\eta$ can be found for general tensor stress state such that a two dimensional model can be extended to three dimensions simply by substituting the two dimensional stress parameters $p_{2 \mathrm{D}}^{\prime}$ and $\eta_{2 D}$ with the general stress parameters $p^{\prime}$ and $\eta$.

The mean effective stress given by equation (6) has been used almost exclusively as the general stress parameter for stress level effect. Then, the focus is to find an appropriate generalized shear stress ratio parameter.

The two methods introduced above can also be classified into this category. For the von Mises method, the shear stress ratio in the deviatoric plane is employed as the general shear stress ratio for the model generalization. For the modified von Mises method, based on the modification on the critical state strength $M(\theta)$ the general shear stress ratio may be defined as follows 


$$
\eta=\frac{q}{p^{\prime}}\left[\frac{M_{2 D}}{M(\theta)}\right]=\frac{3 \sqrt{6}}{2}\left(\frac{\sqrt{\mathbf{s}: \mathbf{s}}}{\operatorname{tr} \boldsymbol{\sigma}^{\prime}}\right)\left[\frac{M_{2 D}}{M(\theta)}\right],
$$

Then, the generalized yield surface for equation (17) can also be written as

$$
f\left(p^{\prime}, \eta, M_{2 D}, p_{o}^{\prime}\right)=f\left[\frac{\operatorname{tr} \boldsymbol{\sigma}^{\prime}}{3}, \frac{3 \sqrt{6}}{2}\left(\frac{\sqrt{\mathbf{s}: \mathbf{s}}}{\operatorname{tr} \boldsymbol{\sigma}^{\prime}}\right)\left[\frac{M_{2 D}}{M(\theta)}\right], M_{2 D}, p_{o}^{\prime}\right]=0
$$

Notice that the manipulation of (17) into (19) is possible only if the yield surface can be written in terms of the normalized shear stress ratio $\eta_{2 D} / M_{2 D}$ for all values of the stress ratio $\eta_{2 D}$ and the critical state strength $M_{2 D}$. It is seen that most constitutive equations for geomaterials satisfy this requirement (e.g., Crouch and Wolf, 1994; Collins and Houlsby, 1997; Potts and Zdravkovic, 1999; Khalili et al, 2005; Dafalias et al, 2006).

The use of the generalized shear stress ratio, other than those described previously under von Mises criterion, is discussed here. The method has been used for extending both constitutive models and constitutive equations (e.g., Lagioia et al, 1996; Gajo and Muir Wood, 1999; Liu and Carter 2004). The generalized stress ratio is normally worked out from a criterion for failure at critical state such as those proposed by Lade (1977), Matsuoka and Nakai (1982) and Liu and Carter (2004). The Matsuoka-Nakai criterion is used here as an example to demonstrate the workings of this method.

Based on the work of Matsuoka and Nakai (1982), the shear stress ratio on a special plane, 
often referred to as the spatially mobilized plane (SMP), is an appropriate stress parameter to describe the effect of stress ratio. The failure surface described by the Matsuoka-Nakai criterion is close to the Mohr-Coulomb failure criterion, and is based on the same critical state friction angle for triaxial compression and extension tests. The general shear stress ratio defined by the criterion is of the form

$\eta_{M N}=\sqrt{\left\{\frac{\operatorname{tr} \boldsymbol{\sigma}^{\prime}\left[\left(t r \boldsymbol{\sigma}^{\prime}\right)^{2}-\left(\boldsymbol{\sigma}^{\prime}: \boldsymbol{\sigma}^{\prime}\right)\right]}{18 J_{3}\left(\boldsymbol{\sigma}^{\prime}\right)}-1\right\}}$,

where $J_{3}$ is given by

$$
J_{3}=\frac{1}{6}\left[2 \operatorname{tr}\left(\boldsymbol{\sigma}^{\prime} \cdot \boldsymbol{\sigma}^{\prime} \cdot \boldsymbol{\sigma}^{\prime}\right)-3 \operatorname{tr} \boldsymbol{\sigma}^{\prime}\left(\boldsymbol{\sigma}^{\prime}: \boldsymbol{\sigma}^{\prime}\right)+\left(\operatorname{tr} \boldsymbol{\sigma}^{\prime}\right)^{3}\right] .
$$

Consequently, the yield surface given by equation (11) will be written as follows for a general stress state

$$
f\left(p^{\prime}, \eta, M_{M N}, p_{o}^{\prime}\right)=f\left[\frac{\operatorname{tr} \boldsymbol{\sigma}^{\prime}}{3}, \sqrt{\left\{\frac{\operatorname{tr} \boldsymbol{\sigma}^{\prime}\left[\left(\operatorname{tr} \boldsymbol{\sigma}^{\prime}\right)^{2}-\left(\boldsymbol{\sigma}^{\prime}: \boldsymbol{\sigma}^{\prime}\right)\right]_{-1}}{18 J_{3}\left(\boldsymbol{\sigma}^{\prime}\right)}, M_{M N}, p_{o}^{\prime}\right]}=0,\right.
$$

It may be noted that in (22) $M_{2 D}$ is replaced by $M_{M N}$, the value of the generalized shear stress ratio $\eta_{M N}$ at critical state. Also notice that, because of the introduction of a new definition of generalized shear stress ratio, $\eta$ may no longer be reduce to $\eta_{2 D}$ for two dimensional stress states. 
This method of model generalization removes the two deficiencies that occur in the modified von Mises method. However, it introduces other difficulties, which have to date remained largely unnoticed in the literature. A brief discussion is given here, and a detailed numerical analysis is presented in Section 5, where a comparison is made between the performance of a model generalized by this method and that by the new method proposed in this study.

One difficulty is that an original two dimensional model cannot be recovered from the generalized model, and that the values of some of the model parameters such as $M$ are not identical to those identified for the original model. This is a major deficiency as the original two dimensional model is usually carefully verified against experimental data and any modifications to it due to the generalization process would be unacceptable.

The second problem is the distortion of material properties in the extension range. For example, an original elliptical yield surface after the generalization may no longer be elliptical even for two dimensional stress states. This can result in the erroneous predictions of the peak strength, the critical state, and stress-strain response to failure.

\subsection{Discussion}

From the literature review, it can be concluded that in geotechnical engineering the stress parameters selected for describing general stress state are predominately the mean effective stress and the shear stress defined by the von Mises criterion. Constitutive models for three dimensional stress and strain states are also predominately formulated based on the von Mises method or modified Mises method. A small percentage of models are 
formulated based on the generalized shear stress ratio derived from failure criterions at critical state for geomaterials (e.g., Lade, 1977; Gajo and Muir Wood, 1999; Liu and Carter; 2004; Matsuoka et al, 1999).

It is widely accepted that the von Mises criterion is not suitable for geomaterials, as it ignores the effect of the third invariant of the stress deviators on the material response. This deficiency has been eliminated in the modified von Mises criterion by rendering $M$ a function of the Lode angle. But the approach introduces the concavity of the yield surface, which poses severe numerical difficulties even at usual range of model parameters. Thus, it can be concluded that the research for model generalization has not been completed, even though a number of advanced approaches have been proposed. Therefore, the proposal of a new method for model generalization, which is simple and in agreement with the experimental data would particularly be useful.

\section{PROPOSED GENERAL METHOD}

A new method for the generalization of two dimensional constitutive models is proposed based on the geometrics of projection and similarity. The three elements for capturing plastic deformation are the yield surface, plastic potential, and the hardening law. The hardening rule, describing the size change of the yield surface with certain internal parameters, usually remains the same for the generalized model. Thus, the work is reduced to the generalization of a two dimensional yield surface and plastic potential into the stress and strain tensor space. 
Although a general tensor stress state is six dimensional, the constitutive relationship can be written in terms of the three stress invariants and the three strain invariants if the stress and strain response of a material is isotropic. Since the three invariants are uniquely determined by the three principal values of the tensor, a general constitutive model can be fully defined in the principal stress and strain space. Two dimensional models are defined in special planes of the principal stress space; that is, planes with two of the three principal stresses being equal. Then, model generalization can be treated as a mathematical manipulation to extend constitutive equations from those special planes to the whole principal stress space.

An additional condition is usually available for the generalization of a surface, that is, the imprint of the surface in another plane (usually a deviatoric plane, i.e., $p^{\prime}=$ constant ). Therefore, surface generalization can be defined as the following mathematical problem.

A general surface in the $\sigma_{1}^{\prime}-\sigma_{2}^{\prime}-\sigma_{3}^{\prime}$ space is sought, the imprints of which are known in the following two perpendicular planes:

1) in the two dimensional $p_{2 D}^{\prime}-q_{2 D}$ plane the surface is defined as $f\left(p_{2 D}^{\prime}, \eta_{2 D}, M_{2 D}, p_{o}^{\prime}\right)=0$, where $M_{2 D}$ is the value of $\eta_{2 D}$ at critical state, and $p^{\prime}{ }_{\mathrm{o}}$ represents the size of the surface, and

2) in a deviatoric plane.

The imprint in the deviatoric plane is usually defined as the intersection of two surfaces. One is the deviatoric plane passing through the point of interests, and the other is a three dimensional surface here denoted by $\rho\left(\sigma_{1}^{\prime}, \sigma_{2}^{\prime}, \sigma_{3}^{\prime}, \xi\right)=0$, in which $\xi$ represents a set of 
parameters controlling the shape of the imprint at the deviatoric plane of interest. The size of the imprint is controlled by the mean effective stress.

A relatively general situation is considered here for the derivation of the general surface (Fig. 4). Suppose that in the principal stress space $\sigma_{1}^{\prime}-\sigma_{2}^{\prime}-\sigma_{3}^{\prime}$, the surface $f\left(p_{2 D}^{\prime}, \eta_{2 D}, M_{2 D}, p_{o}^{\prime}\right)=0$ and the imprint in the deviatoric plane have experienced translation $\mathbf{c}=\left(c_{1}, c_{2}, c_{3}\right)$, and rotation $\gamma=\left(\gamma_{1}, \gamma_{2}, \gamma_{3}\right)$. To solve this problem, the expression of the general surface in the local coordinates $\sigma_{1}^{\prime \prime}-\sigma_{2}^{\prime \prime}-\sigma_{3}^{\prime \prime}$, where the surface has no translation or rotation, is determined first. Then, its expression in the global coordinates is derived. Parameters in the local coordinates are indicated by symbol ".

Now, suppose in the local coordinates $\sigma_{1}^{\prime \prime}-\sigma_{2}^{\prime \prime}-\sigma_{3}^{\prime \prime}$ the two dimensional surface is defined by $f\left(p_{2 D}^{\prime \prime}, \eta_{2 D}^{\prime \prime}, M_{2 D}, p_{o}^{\prime}\right)=0$, and the imprint in the local deviatoric plane at point A (Fig. 4) is given as

$$
\left\{\begin{array}{l}
\rho\left(\sigma_{1}^{\prime \prime}, \sigma_{2}^{\prime \prime}, \sigma_{3}^{\prime \prime}, \xi\right)=0 \\
p^{\prime \prime}=p_{2 D A}^{\prime \prime}
\end{array}\right.
$$

Through the operation represented by equation (23), point A is, in essence, generalized into a curve in the local deviatoric plane. The proposed method of model generalization is simply to define the generalized curve in the deviatoric plane for any given stress state on the surface $f\left(p_{2 D}^{\prime \prime}, \eta_{2 D}^{\prime \prime}, M_{2 D}, p_{o}^{\prime}\right)=0$, by using the operation represented by equation (23). This is achieved through a linear translation of $\rho\left(\sigma_{1}^{\prime \prime}, \sigma_{2}^{\prime \prime}, \sigma_{3}^{\prime \prime}, \xi\right)=0$ along $p^{\prime \prime}$ axis such that 
function $\rho\left(\sigma_{1}^{\prime \prime}-k p^{\prime \prime}, \sigma_{2}^{\prime \prime}-k p^{\prime \prime}, \sigma_{3}^{\prime \prime}-k p^{\prime \prime}, \xi\right)=0$ passes through the stress point of interest. $k$ is the translation coefficient. Graphical representation of the technique for stress point B is shown in Fig. 4. The following curve is obtained for the stress point B.

$$
\left\{\begin{array}{l}
\rho\left(\sigma_{1}^{\prime \prime}-k p^{\prime \prime}, \sigma_{2}^{\prime \prime}-k p^{\prime \prime}, \sigma_{3}^{\prime \prime}-k p^{\prime \prime}, \xi\right)=0 \\
p^{\prime \prime}=p_{2 D B}^{\prime \prime}
\end{array}\right.
$$

The collection of the imprints for all stress points on the two dimensional surface yields the generalized surface in the local stress space. The generalization may be performed homothetically or non-homothetically by keeping $\xi$ constant or varying it as a function of $p^{\prime \prime}$ or other state parameters, as appropriate. The homothetic translation will be relevant in soil engineering, where the deviatoric cross sections of the generalized surface are often geometrically similar. The non-homothetic translation may be appropriate for materials such as concrete and rock, in which the deviatoric cross section of the yield surface becomes more circular with increasing mean effective stress.

The translation parameter $k$ is quantified by insisting that the stress point of interest satisfies the function $\rho\left(\sigma_{1}^{\prime \prime}-k p^{\prime \prime}, \sigma_{2}^{\prime \prime}-k p^{\prime \prime}, \sigma_{3}^{\prime \prime}-k p^{\prime \prime}, \xi\right)=0$. In geomechanics, the imprint of the three dimensional surface in the deviatoric plane is usually defined at the critical state using the failure criterion, which for frictional materials is represented by a linear function passing through the origin of the coordinates, $\rho\left(\sigma_{1}^{\prime \prime}, \sigma_{2}^{\prime \prime}, \sigma_{3}^{\prime \prime}, M_{2 D}\right)=0$. A study of the shape for such surfaces can be found in a paper by Liu and Carter (2003). In this case, $k$ is readily obtained from the geometrics of the problem (Fig. 5) and the generalized surface in the local coordinate system takes the form, 


$$
\left\{\begin{array}{c}
\rho\left(\sigma_{1}^{\prime \prime}-k p^{\prime \prime}, \sigma_{2}^{\prime \prime}-k p^{\prime \prime}, \sigma_{3}^{\prime \prime}-k p^{\prime \prime}, M_{2 D}\right)=0 \\
k=1-\frac{\eta_{2 D}^{\prime \prime}}{M_{2 D}}=1-\frac{\eta_{2 D}^{\prime \prime}\left(p_{2 D}^{\prime \prime}, M_{2 D}, p_{o}^{\prime}\right)}{M_{2 D}}
\end{array} .\right.
$$

in which $\eta_{2 D}^{\prime \prime}$ is the stress ratio on $f\left(p_{2 D}^{\prime \prime}, \eta_{2 D}^{\prime \prime}, M_{2 D}, p_{o}^{\prime}\right)=0$, obtained from the rearrangement $f\left(p_{2 D}^{\prime \prime}, \eta_{2 D}^{\prime \prime}, M_{2 D}, p_{o}^{\prime}\right)=0$ as $\eta_{2 D}^{\prime \prime}=\eta_{2 D}^{\prime \prime}\left(p_{2 D}^{\prime \prime}, M_{2 D}, p_{o}^{\prime}\right)$.

With the mathematical form for the generalized surface in the local coordinates determined, its form in the global coordinates can be determined by standardized operators. The translation of the surface is described by

$$
\left\{\begin{array}{l}
\sigma_{1}^{\prime \prime}=\sigma_{1}^{\prime}-c_{1} \\
\sigma_{2}^{\prime \prime}=\sigma_{2}^{\prime}-c_{2} \\
\sigma_{3}^{\prime \prime}=\sigma_{3}^{\prime}-c_{3}
\end{array}\right.
$$

Substituting equation (26) into (25), the general surface in the principal stress space is obtained. The application of the translated yield surface is seen in a number of situations such as modeling the effect of cementation, the kinematic hardening effect, and the effect of suction (e.g; Kavvadas and Amorosi, 2000; Khalili et al, 2005).

The effect of rotation of the yield surface mainly results from rotational hardening of the material. The rotation hardening proposed by Sekiguch and Ohta (1977) is widely used in geomechanics and is also adopted for this research (e.g., Pestana and Whittle, 1999; Gajo 
and Muir Wood, 2001). According to Sekiguchi-Ohta's proposal, during rotational hardening all the deviatoric components of a stress state are decreased by $p^{\prime} \gamma$, where $\gamma$ is the rotational hardening parameter tensor, meanwhile the value of the mean effective stress remains unchanged. As a result, the combined effect of rigid translation and rotation is described by

$$
\left\{\begin{array}{l}
\sigma_{1}^{\prime \prime}=\sigma_{1}^{\prime}-c_{1}-\left(p^{\prime}-\frac{c_{1}+c_{2}+c_{3}}{3}\right) \gamma_{1} \\
\sigma_{2}^{\prime \prime}=\sigma_{2}^{\prime}-c_{2}-\left(p^{\prime}-\frac{c_{1}+c_{2}+c_{3}}{3}\right) \gamma_{2} \\
\sigma_{3}^{\prime \prime}=\sigma_{3}^{\prime}-c_{3}-\left(p^{\prime}-\frac{c_{1}+c_{2}+c_{3}}{3}\right) \gamma_{3}
\end{array}\right.
$$

Written in terms of tensors, the above relationship can be expressed as

$$
\boldsymbol{\sigma}^{\prime \prime}=\boldsymbol{\sigma}^{\prime}-\mathbf{c}-\left(p^{\prime}-\frac{\operatorname{trc}}{3}\right) \gamma
$$

It may be noticed that the rotational hardening proposed by Sekiguch and Ohta (1977) introduces distortion of the surface. For an example, with the occurrence of rotational hardening an original elliptical yield surface is no longer elliptical.

Of course, when $\mathbf{c}$ and $\gamma$ are null, then local and global coordinates coincide and the generalize surface takes the form 


$$
\left\{\begin{array}{c}
\rho\left(\sigma_{1}^{\prime}-k p^{\prime}, \sigma_{2}^{\prime}-k p^{\prime}, \sigma_{3}^{\prime}-k p^{\prime}, M_{2 D}\right)=0 \\
k=1-\frac{\eta_{2 D}^{\prime}}{M_{2 D}}=1-\frac{\eta_{2 D}^{\prime}\left(p_{2 D}^{\prime}, M_{2 D}, p_{o}^{\prime}\right)}{M_{2 D}}
\end{array}\right.
$$

\section{GENERALIZATION OF TWO DIMENSIONAL CONSTITUTIVE MODELS}

The proposed method can be employed quite easily for generalizing an existing two dimensional model. A detailed example is given below. The well known Modified Cam Clay model is chosen which has a physical basis and is widely used in geotechnical engineering research. For comparison, the model is also generalized using the generalized shear stress ratio method discussed in Section 2.3.

\subsection{Summary of the Modified Cam Clay model}

Details of the Modified Cam Clay (MCC) model can be found in Muir Wood (1990). The main features of the model are summarized here.

\subsubsection{Yield surface and plastic potential}

The yield surface is assumed to be elliptical in shape (Fig. 6). In terms of the mean effective stress and the shear stress ratio it is expressed as

$$
f=p_{o}^{\prime}-\left[1+\left(\frac{\eta_{2 D}}{M_{2 D}}\right)^{2}\right] p_{2 D}^{\prime}=0
$$


in which $p^{\prime}{ }_{o}$ represents the size of the yield surface. $M_{2 D}$ controls the aspect ratio of the yield surface, and is related to the critical state friction angle $\phi_{c s}$ using

$$
M_{2 D}=\frac{6 \sin \phi_{c s}}{3-\sin \phi_{c s}}
$$

Associated plastic flow is assumed so that the yield surface is also the plastic potential, $g$, i.e.

$$
g=f
$$

The plastic flow rule is defined using the dilatancy relationship

$$
\frac{d \varepsilon_{d, 2 D}^{p}}{d \varepsilon_{v, 2 D}^{p}}=\frac{2 \eta_{2 D}}{\left(M_{2 D}^{2}-\eta_{2 D}^{2}\right)} .
$$

Superscript $p$ is used for plastic deformation.

\subsubsection{Hardening law}

Hardening is governed by the plastic volumetric strain, such that

$\frac{d p_{o}^{\prime}}{p_{o}^{\prime}}=\frac{v}{(\lambda-\kappa)} d \varepsilon_{v, 2 D}^{p}$, 
where $v$ is the specific volume, $\lambda$ is the virgin compression index, and $\kappa$ is the elastic swelling and recompression index.

\subsubsection{Stress and strain relationships}

The incremental stress and strain relationships are in turn written as,

$$
\begin{aligned}
& d \varepsilon_{v, 2 D}=d \varepsilon_{v, 2 D}^{e}+(\lambda-\kappa) \frac{d p_{o}^{\prime}}{v p_{o}^{\prime}}, \\
& d \varepsilon_{d, 2 D}=d \varepsilon_{d, 2 D}^{e}+\frac{2 \eta_{2 D}(\lambda-\kappa)}{\left(M_{2 D}^{2}-\eta_{2 D}^{2}\right)} \frac{d p_{o}^{\prime}}{v p_{o}^{\prime}} .
\end{aligned}
$$

in which, superscript $e$ is used for elastic deformation.

\subsection{Modified Cam Clay model generalized via the proposed method}

The general Modified Cam Clay (MCC) model extended via the proposed method is introduced in this section. The Matsuoka-Nakai criterion is selected to represent the critical state surface for geomaterials. In the MCC model, the effect of kinematic hardening and rotational hardening are not considered; thus, only the forms of yield surface and plastic potential in a global coordinates are considered. 


\subsubsection{Elastic deformation}

The elastic deformation in the general stress and strain states is assumed to obey Hook's law. It follows that the elastic strain increment tensor is linked to the stress increment tensor as,

$d \varepsilon^{e}=\frac{(1+v) d \boldsymbol{\sigma}^{\prime}}{E}-\frac{v \operatorname{tr}\left(d \boldsymbol{\sigma}^{\prime}\right)}{E} \boldsymbol{\delta}$,

where $E$ is the Young's modulus and is related to Poisson's $v$, the elastic swelling index $\kappa$, specific volume $v$, and the mean effective stress $p^{\prime}$ as

$$
E=\frac{3(1-2 v) v}{\kappa} p^{\prime}
$$

\subsubsection{Generalized yield surface and plastic potential}

To generalize the yield surface, we first re-arrange the elliptical yield surface (30) as

$$
\eta_{2 D}=M_{2 D}\left(\sqrt{\frac{p_{o}^{\prime}}{p_{2 D}^{\prime}}-1}\right)
$$

Matsuoka-Nakai criterion (1982) is selected to represent imprint of the critical state surface in the deviatoric plane, which in terms of the general stress tensor is written as 
$\rho\left(\boldsymbol{\sigma}^{\prime}, \phi_{c s}\right)=9\left\{\frac{\operatorname{tr} \boldsymbol{\sigma}^{\prime}\left[\left(t r \boldsymbol{\sigma}^{\prime}\right)^{2}-\boldsymbol{\sigma}^{\prime}: \boldsymbol{\sigma}^{\prime}\right]_{-1}}{18 J_{3}\left(\boldsymbol{\sigma}^{\prime}\right)}\right\}-8\left(\tan \phi_{c s}\right)^{2}=0$

where $J_{3}\left(\boldsymbol{\sigma}^{\prime}\right)$ is the third stress invariant and is given by equation (21). Matsuoka-Nakai criterion is a linear cone passing through the origin of the principal stress space; thus, the general yield surface in the stress tensor space can simply be obtained as,

$$
\left\{\begin{array}{l}
9\left\{\frac{\operatorname{tr}\left(\boldsymbol{\sigma}^{\prime}-k p \boldsymbol{\delta}\right)\left\{\left[\operatorname{tr}\left(\boldsymbol{\sigma}^{\prime}-k p \boldsymbol{\delta}\right)\right]^{2}-\left(\boldsymbol{\sigma}^{\prime}-k p \boldsymbol{\delta}\right):\left(\boldsymbol{\sigma}^{\prime}-k p \boldsymbol{\delta}\right)\right\}}{18 J_{3}\left(\sigma^{\prime}-k p \delta\right)}-1\right\}-8\left(\tan \varphi_{c s}\right)^{2}=0 \\
k=1-\frac{\eta_{2 D}}{M_{2 D}}=1-\left(\sqrt{\frac{p_{o}^{\prime}}{p^{\prime}}-1}\right)
\end{array}\right.
$$

The value of the mean effective stress $p^{\prime}$ varies from 0 to $p_{o}^{\prime} . \quad J_{3}\left(\boldsymbol{\sigma}^{\prime}-k p \boldsymbol{\delta}\right)$ is computed according to

$$
\begin{aligned}
& J_{3}\left(\boldsymbol{\sigma}^{\prime}\right)=\left(\sigma_{1}^{\prime}-k p^{\prime}\right)\left(\sigma_{2}^{\prime}-k p^{\prime}\right)\left(\sigma_{3}^{\prime}-k p^{\prime}\right)=\frac{1}{6} \times \\
& \left\{2 \operatorname{tr}\left[\left(\boldsymbol{\sigma}^{\prime}-k p \boldsymbol{\delta}\right) \cdot\left(\boldsymbol{\sigma}^{\prime}-k p \boldsymbol{\delta}\right) \cdot\left(\boldsymbol{\sigma}^{\prime}-k p \boldsymbol{\delta}\right)\right]-3 \operatorname{tr}\left(\boldsymbol{\sigma}^{\prime}-k p \boldsymbol{\delta}\right)\left[\left(\boldsymbol{\sigma}^{\prime}-k p \boldsymbol{\delta}\right):\left(\boldsymbol{\sigma}^{\prime}-k p \boldsymbol{\delta}\right)\right]+\left[\operatorname{tr}\left(\boldsymbol{\sigma}^{\prime}-k p \boldsymbol{\delta}\right)\right]^{3}\right\}
\end{aligned}
$$

Associated flow rule is assumed. The plastic potential and the yield surface are identical.

\subsubsection{Plastic deformation}

The general expression for the plastic deformation is described by 


$$
d \boldsymbol{\varepsilon}^{p}=\Lambda \frac{\partial g}{\partial \boldsymbol{\sigma}^{\prime}}
$$

The hardening function remains the same, as given by equation (34). As a result, the multiplier $\Lambda$ can be determined from the hardening function. The plastic deformation is obtained as

$$
d \boldsymbol{\varepsilon}^{p}=\frac{(\lambda-\kappa)}{v}\left(\frac{d p_{o}^{\prime}}{p_{o}^{\prime}}\right) \frac{\frac{\partial g}{\partial \boldsymbol{\sigma}^{\prime}}}{\operatorname{tr}\left(\frac{\partial g}{\partial \boldsymbol{\sigma}^{\prime}}\right)}
$$

\subsubsection{The stress and strain relationship}

Satisfying the consistency condition for the generalized yield surface, the following incremental stress and strain relationship is obtained

$$
d \boldsymbol{\varepsilon}=\frac{(1+v) d \boldsymbol{\sigma}^{\prime}}{E}-\frac{\nu \operatorname{trd} \boldsymbol{\sigma}^{\prime}}{E} \boldsymbol{\delta}+\frac{(\lambda-\kappa)}{v}\left(\frac{d p_{o}^{\prime}}{p_{o}^{\prime}}\right) \frac{\frac{\partial g}{\partial \boldsymbol{\sigma}^{\prime}}}{\operatorname{tr}\left(\frac{\partial g}{\partial \boldsymbol{\sigma}^{\prime}}\right)}
$$

\subsection{Modified Cam Clay model extended via generalized shear stress ratio}

For the purpose of comparison, the Modified Cam Clay model is also generalized using the "generalized" shear stress ratio method detailed in Section 2.3. The general mean effective for tensor stress state is given by equation (6). Matsuoka-Nakai criterion is also adopted 
for the definition of the general shear stress ratio. Thus, the general shear stress ratio is given by equation (20). The following generalized equations are then obtained through a simple substitution of $\eta_{2 D}$ with $\eta_{M N}$.

The yield surface and the plastic potential are given by

$$
f=g=p_{o}^{\prime}-\left[1+\left(\frac{\eta_{M N}}{M_{M N}}\right)^{2}\right] p^{\prime}=0
$$

in which, $M_{M N}$ is the value of the stress ratio at critical state and can be expressed in terms of the critical state friction angle measured from conventional triaxial compression test, $\phi_{c s}$,

$$
M_{M N}=\frac{2 \sqrt{2}}{3} \tan \phi_{c s}
$$

The hardening function is the same as the original Modified Cam Clay model, given by equation (34). The elastic strain is described by Hook's law, is independent of the method for model generalization, and is given by equation (37). Consequently, the incremental stress and strain relationship is identical to that in equation (45). 


\section{APPLICATION}

In order to evaluate the applicability of the proposed method for model generalization, the main features of the generalized Modified Cam Clay model are examined and the model is used to simulate the drained behaviour of a single soil element for loading in the principal stress space. Results from parallel simulations, using the model formulated based on the generalized shear stress ratio method, are also presented for the sake of comparison.

For loading in the principal stress space, parameter $b$ is often used as an indicator of the relative magnitude of the intermediate stress and is defined as

$$
b=\frac{\sigma_{2}^{\prime}-\sigma_{3}^{\prime}}{\sigma_{1}^{\prime}-\sigma_{3}^{\prime}} .
$$

Constant $b$ refers to all stress states that remain on one plane passing through the isotropic stress state line. The intersection of this plane with the deviatoric plane is a radial line from the origin of the coordinates. In all the simulations, one set of soil parameters are adopted, as listed in Table 1.

Table 1 Model Parameters

\begin{tabular}{|c|c|c|c|c|c|}
\hline Parameter & $\phi_{c s}$ & $\lambda$ & $\kappa$ & $e_{I C}$ & $v$ \\
\hline value & $30^{\circ}$ & 0.16 & 0.05 & 2.176 & 0.25 \\
\hline
\end{tabular}

In Table $1, e_{I C}$ is the void ratio at $p^{\prime}=1 \mathrm{kPa}$ on the isotropic compression line (ICL). 


\subsection{Basic features of the generalized models}

For both versions of the generalized Modified Cam Clay model, the intersection of the yield surfaces with the planes of constant $b$ are shown in Figs 7 and 8. Three values of $b$ are considered. They are: $\sigma_{1}^{\prime}>\sigma_{2}^{\prime}=\sigma_{3}^{\prime} \quad(b=0), \quad \sigma_{2}^{\prime}=0.5\left(\sigma_{1}^{\prime}+\sigma_{3}^{\prime}\right) \quad(b=0.5), \quad$ and $\sigma_{1}^{\prime}=\sigma_{2}^{\prime}>\sigma_{3}^{\prime}(b=1) . p_{\mathrm{o}}^{\prime}$ is taken as $100 \mathrm{kPa}$.

It is seen from Fig. 7 that the yield surfaces generated using the proposed method are elliptical for various intersections with planes of constant $b$. The summits of the ellipses remain on one plane with $p^{\prime}=0.5 p_{o}^{\prime}$. The critical state of deformation is reached when the stress state reaches the summit of the yield surface, where shear stress ratio is equal to the value defined by critical state surface. In contrast, the yield surfaces obtained by the generalized shear stress ratio method are not elliptical and differ substantially from the original two dimensional yield surface (Fig. 8). More importantly, the summits of these surfaces do not occur at $p^{\prime}=0.5 p_{o}^{\prime}$, nor are on a plane. As can be seen from equation (46), soil on the yield surface reaches the critical state stress ratio at $p^{\prime}=0.5 p_{o}^{\prime}$. Thus, if associated flow rule is assumed, the Modified Cam Clay model extended through this method cannot predict the failure state of material on the yield surface using the input critical state stress ratio. This is in contradiction with the characteristic of the Modified Cam Clay model. This difficulty is also shown in the $\sigma_{1}^{\prime} / \sigma^{\prime}{ }_{3}$ versus shear stress ratio response obtained for the conventional triaxial compression test predicted using the two generalized models (Fig. 9). The general shear stress ratio via the proposed method 
and $\eta_{2 D}$ are identical, but the stress ratio via the generalized shear stress ratio method and $\eta_{2 D}$ are different and their relationship is nonlinear. As a result, the yield surface via this generalization does not remain elliptical, and is distorted.

For both methods, the shape of yield surface in the deviatoric plane is that described by Matsuoka-Nakai criterion, which is unconditionally convex. Therefore, the general yield surface and plastic potential are also convex.

\subsection{Behaviour of soils for loading in the principal stress space}

To further investigate the application of the proposed generalization technique, drained tests in principal stress space are simulated. The stress path for the tests is linear in the deviatoric plane, as shown in the insert in Fig. 10, with parameter $b$ varying from 0 to 1 , where the intermediate principal stress $\sigma_{2}^{\prime}$ varies from being equal to the minimum principal stress to being equal to the maximum principal stress. Three tests are simulated with $b=0,0.5$, and 1 . For all the tests, the value of the mean effective stress is kept constant at $100 \mathrm{kPa}$. The initial state of the soil is assumed to be on the tip of the yield surface corresponding to $p_{o}^{\prime}=100 \mathrm{kPa}$. Thus, all soil specimens exhibit virgin yielding during shearing.

The simulations made using the proposed method, presented in terms of shear stress versus principal strain, are shown in Figs 10 to 12 . The general shear stress $q_{V M}$ corresponding to von Mises criterion is used in the presentations, which represents the deviation of the stress state from the isotropic condition. The stress ratio versus distortional strain relationship is 
given in Fig. 13 and the distortional and volumetric strain relationship is shown in Fig. 14. For the simulations made using the generalized shear stress ratio method, the shear stress and principal strain relationship is shown in Figs 15 to 17 . The stress ratio and distortional strain relationship is shown in Fig. 18.

It can be seen that the pattern of the variation of the three principal strains simulated via the proposed method is consistent with experimental observation for both clays and sands, (e.g., Yamada, 1979; Nakai and Hinokio, 2004; Lade and Musante, 1978). The following main features may be noted:

1) For a soil in a loose state, i.e., on the wet side of the critical state, soil hardens steadily until failure is reached. There is only one strength for the soil, the critical state strength. For this type of behaviour, the volumetric deformation is compressive.

2) The direction of plastic strain increment vector in the deviatoric plane is approximately normal to the failure criterion of the soil.

3) The volumetric and distortional strain relationship for tests, with linear stress paths in the deviatoric plane, with different values of $b$ is close, but not necessarily coincident.

It can also be seen that the deformation of soil in the principal stress and strain space is not represented satisfactorily by the model extended by the generalized shear stress ratio method. The final failure strength predicted does not correspond to the input critical state strength parameters of the test soil. As seen in Fig. 18, the final failure stress ratios predicted for triaxial compression and extension tests are 2.8 and 2.3 respectively. However, the critical state stress ratio for triaixal tests is 3 for $\phi_{c s}=30^{\circ}$. 
In general, the results show that constitutive models extended by the generalized shear stress ratio method may fail to provide reasonable simulation of soil behaviour in the general stress space. Through the generalization, the original elliptical surface is distorted, even for the axially symmetrical stress states. Consequently, contradictions occur rendering the model unable to predict the soil response based on the actual parameters of the soil. In particular, summits of the surfaces at different value of Lode angle are deviated out of plane. This type of distortion in the generalized three dimensional surfaces has been observed in some of the pervious works reported in the literature, with similar consequences. Examples include, the yield surfaces in the family of constitutive models developed by Dean (1998) from very innovative approach based on the so called specific length concept, the yield surface in a conceptual model for clays developed by Houlsby and Sharma (1999) based on micromechanical consideration, and the yield surface developed by Matsuoka et al (1999) for model generalization based on a scaled translation technique. Such deficiencies are removed using the method proposed.

\section{CONCLUSIONS}

Most constitutive models for geomaterials under general stress and strain tensor conditions are developed from two dimensional models. Existing methods of model generalization are reviewed and their limitations are highlighted. A simple and standardized method for model generalization has been proposed based on the imprints of the generalized surface on two perpendicular planes: the original surface in the $p_{2 D}^{\prime}-q_{2 D}$ space and the imprint of the surface in one deviatoric plane. Equations for the generalization of a surface are 
derived. The workings of the proposed method are demonstrated by generalizing the Modified Cam Clay model using the Matsuoka-Nakai criterion at the critical state. It is seen that the generalized Modified Cam Clay model has the capacity to represent the behaviour of soil in the general stress and strain space. Deficiencies in the existing methods for model generalization have been removed and the proposed method has been shown to possess the following advantages: (1) the general model is identical to the original model for axially symmetrical stress and strain states, (2) the critical state of geomaterials can be accurately described without altering the input soil parameters, and (3) the convexity of the yield surface and plastic potential in the general stress space are ensured provided the imprints of the generalized surface utilized in the model generalization are convex.

\section{REFERENCES}

Arduino P. and Macari E. J. (2001), "Implementation of porous medial formulation for geomaterials", Journal of Engineering Mechanics, ACME, Vol. 127(2), pp.157-166.

Argyris J. H., Faust G., Szimat J., Warnke P., and William K. (1974), "Recent developments in finite element analyses of prestressed concrete reactor vessels", Nuclear Engineering and Design, Vol. 28, pp.42-75.

Callisto and Calabresi (1998), "Mechanical behaviour of a natural soft clay", Géotechnique, Vol. 48(4), pp. 495-513.

Collins I. F. and Houlsby G. T. (1997), “Application of the thermomechanical principles to the modeling of geotechnical materials", Proc. Royal Society, London A, Vol. 453, pp.1975-2001. 
Crouch RS, Wolf JP. (1994). Unified 3D critical state bounding-surface plasticity model for soils incorporating continuous plastic loading under cyclic paths. Part I: constitutive relations. International J. of Numerical and Analytical Methods in Geomechanics, Vol. 18 , pp.735-758.

Dean E. T. R. (1998), "Specific length and some constitutive models", Géotechnique, Vol. 48(1), pp.1-32.

Dafalias Y. F., Manzari, M. T., and Papadimitriou A. G. (2006), "Saniclay: simple anisotropic clay plasticity model”, International J. for numerical and analytical methods in Geomechanics, Vol. 30, pp.1231-1257.

Gajo A. and Muir Wood D. (1999), “A kinematic hardening constitutive model for sands: the multiaxial formulation”, Int. J. for Numerical and Analytical Methods in Geomechanics, Vol. 23, pp.925-964.

Gajo A. and Muir Wood D. (2001), "A new approach to anisotropic, bounding surface plasticity: general formulation and simulation of natural and reconstituted clay behaviour", Int. J. for Numerical and Analytical Methods in Geomechanics, Vol. 25, pp.207-241.

Houlsby G. T. and Sharma R. S. (1999), "A conceptual model for the yielding and consolidation of clays", Géotechnique, Vol. 49 (4), pp.491-501.

Kavvadas M. and Amorosi A. (2000), “A constitutive model for structured soils", Géotechnique, Vol. 50(3), pp.263-273.

Khalili N., Habte M. A. and Valliappan S. (2005), “A bounding surface plasticity model for cyclic loading of granular soils", International J. for Numerical and Analytical Methods in Geomechanics. Vol 63, pp.1939-1960.

Lade P. V. (1977), "Elastoplastic stress-strain theory for cohesionless soil with curved yield surfaces", International Journal of Solids and Structures, Vol. 13, No. 1, pp. 63-76. 
Lade P. V. and Musante H. M. (1978), “Three-dimensional behaviour of remoulded clay”, $J$. Geotechnical Engineering, ASCE, Vol. 104(2), pp.193-209.

Lagioia R., Puzrin A. M., and Potts D. M. (1996), "A new versatile expression for yield and plastic potential surfaces", Computers and Geotechnics, Vol. 19(3), pp.171-191.

Liu M. D. and Carter J. P. (2002), "Structured Cam Clay Model”, Canadian Geotechnical J.. Vol. 39(6), 1313-1332.

Liu M. D. and Carter J. P. (2003), “A general strength criterion for geo-materials", International J. of Geomechanics, ASCE, Vol 3(3/4), pp.253-259.

Liu M. D. and Carter J. P. (2004), "Evaluation of the Sydney Soil Model”, Advances in Geotechnical Engineering: The Skempton Conference, London, Vol 1, pp.498-509.

Matsuoka H. and Nakai T. (1982), "A new failure criterion for soils in three dimensional stress", Proc. of conference on deformation and failure of granular materials, Delft, Vermeer et al (ed), pp.253-263.

Matsuoka H., Yao Y., and Sun D. (1999), "The Cam-Clay models revised by the SMP criterion”, Soils and Foundations, Vol. 39(1), pp.81-95.

Muir-Wood D. (1990), Soil Behaviour and Critical State Soil Mechanics, Cambridge University Press.

Nakai T. and Mihara Y. (1984), “A new mechanical quantity for soils and its application to elastoplastic constitutive models", Soils and Foundations, Vol. 24(2), pp.82-94.

Nakai T. and Hinokio M. (2004), "A simple elastoplastic model for normally and over consolidated soils with unified material parameters", Soils and Foundations, Vol. 44(2), pp.53-70.

Pestana J. M. and Whittle A. J. (1999), "Formulation of a unified constitutive model for clay and sands", Int. J. for Numerical and Analytical Methods in Geomechanics. Vol. 23, pp.1215-1243. 
Potts D. M. (2003), “Numerical analysis: a virtual dream or practical reality?", Géotechnique, Vol. 53(6), pp.535-573.

Potts D. M. and Zdravkovic L. (1999), Finite Element Analysis in Geotechnical Engineering: Theory, London, Thomas Telford.

Sekiguch H. and Ohta H. (1977), "Induced anisotropy and time dependency in clays", Proc. $9^{\text {th }}$ Int. Conference Soil Mechanics and Foundation Engineering, pp.229-238.

Sheng D., Sloan S. W. and Yu H. S. (2000), “Aspects of finite element implementation of critical state models", Computational Mechanics, Vol. 26(2), pp.185-196.

Tsutsumi S. and Hashiguchi K. (2005), “General non-proportional loading behaviour of soils”, International Journal of Plasticity, Vol. 21, pp.1941-1969.

Wheeler S. J., Naatanen A., Karstunen M., and Lojander M. (2003), “An anisotropic elastoplastic model for soft clays”, Canadian Geotechnical J., Vol. 40(2), pp.403-418.

Yamada Y. (1979), Deformation Characteristics of Loose Sand Under Three Dimensional Stress Conditions, Ph.D. thesis, Tokyo University. 


\section{List of Figures:}

Figure 1: Generalization using von Mises method

Figure 2: Failure surface in deviatoric plane according to von Mises, Mohr Coulomb and typical of those encountered for geomaterials.

Figure 3: Failure surface in deviatoric according to equation (15) for $\phi_{c s}=30^{\circ}$ and

$$
\phi_{c s}=40^{\circ}
$$

Figure 4: Schematic representation of proposed generalization technique in the general stress space with a non-linear operator.

Figure 5: Schematic representation of proposed generalization technique in the local coordinate system with a linear operator.

Figure 6: Modified Cam Clay model.

Figure 7: Intersections of the yield surface with different $b$ for the proposed model.

Figure 8: Intersections of the yield surface for the model extended via generalized shear stress ratio.

Figure 9: Generalized shear stress ratios via various methods for axysymmetical stress states.

Figure 10: Principal strains simulated via the proposed method for a test with $b=0\left(\beta=0^{\circ}\right)$.

Figure 11: Principal strains simulated via the proposed method for a test with $b=0.5$ $\left(\beta=30^{\circ}\right)$.

Figure 12: Principal strains simulated via the proposed method for a test with $b=1\left(\beta=60^{\circ}\right)$.

Figure 13: Shear stress ratio and distortional strain relationship simulated via the proposed method.

Figure 14: Volumetric and distortional strain relationship simulated via the proposed 
method.

Figure 15: Principal strains simulated via the generalized stress ratio method for a test with $b=0\left(\beta=0^{\circ}\right)$.

Figure 16: Principal strains simulated via the generalized stress ratio method for a test with $b=0.5\left(\beta=30^{\circ}\right)$.

Figure 17:Principal strains simulated via the generalized stress ratio method for a test with $b=1\left(\beta=60^{\circ}\right)$.

Figure 18: Shear stress ratio and distortional strain relationship simulated via the generalized shear stress ratio method. 


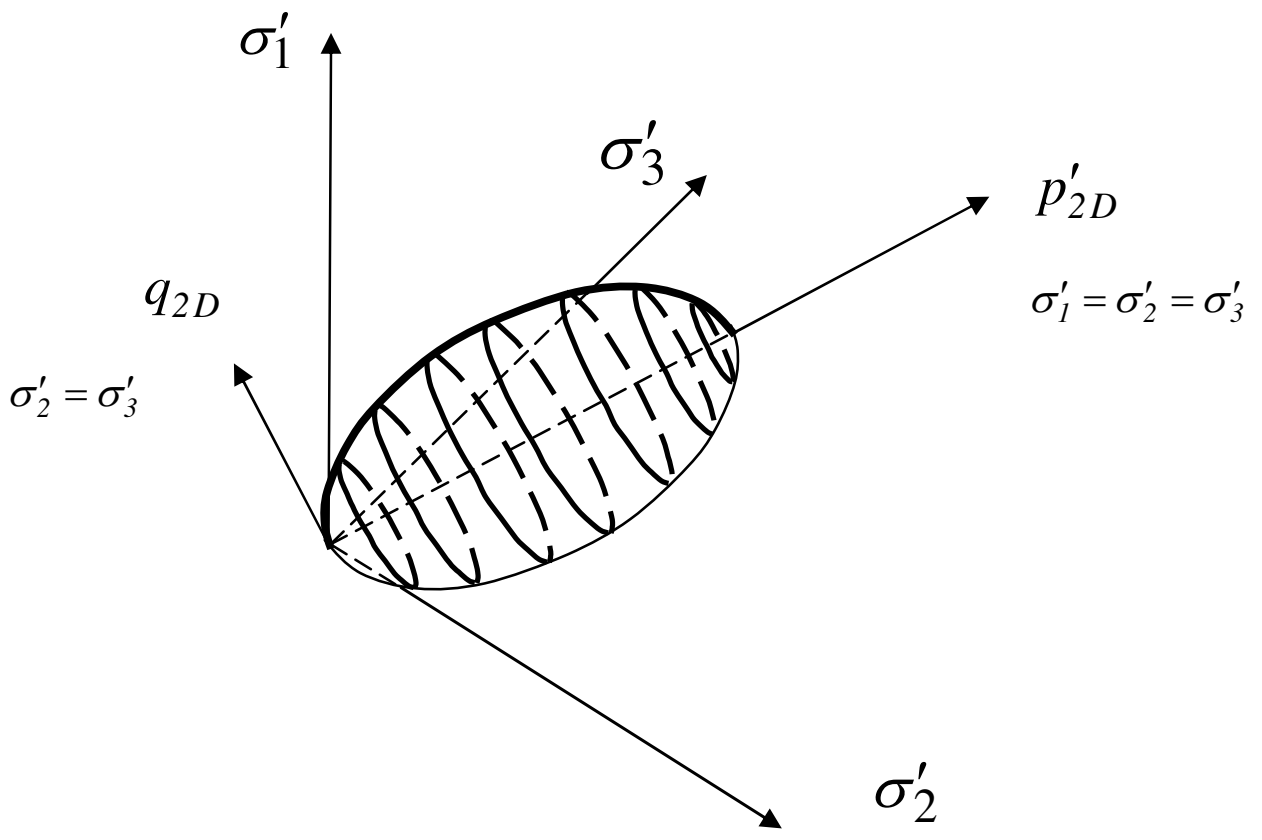

Figure 1: Generalization using von Mises method 


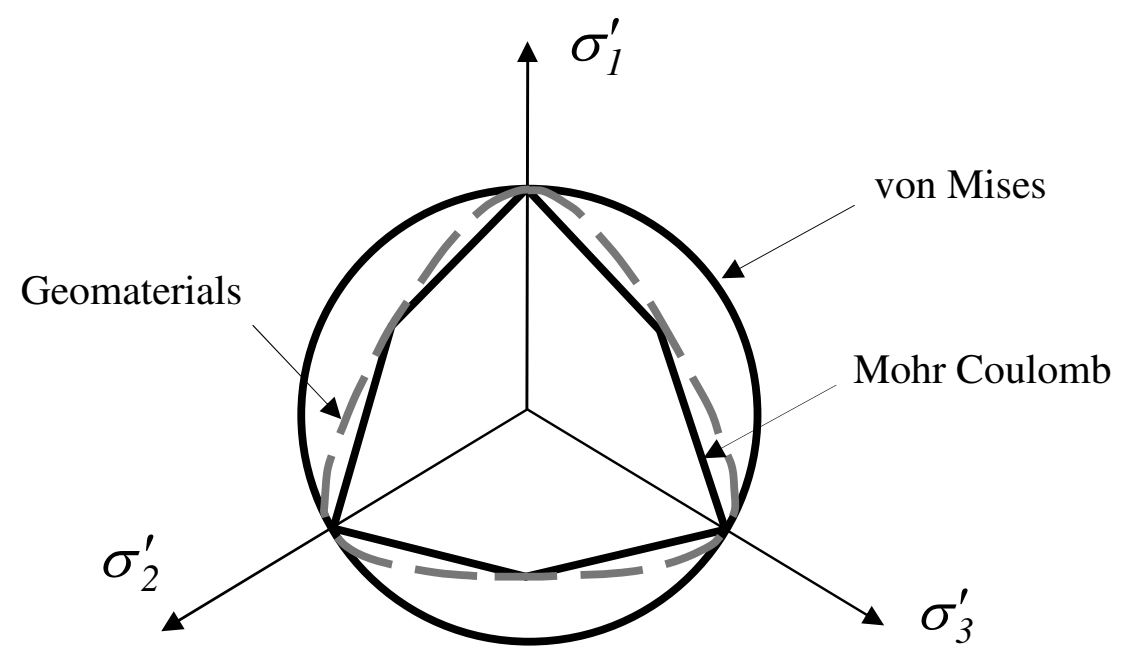

Figure 2: Failure surface in deviatoric plane according to von Mises, Mohr Coulomb and typical of those encountered for geomaterials. 

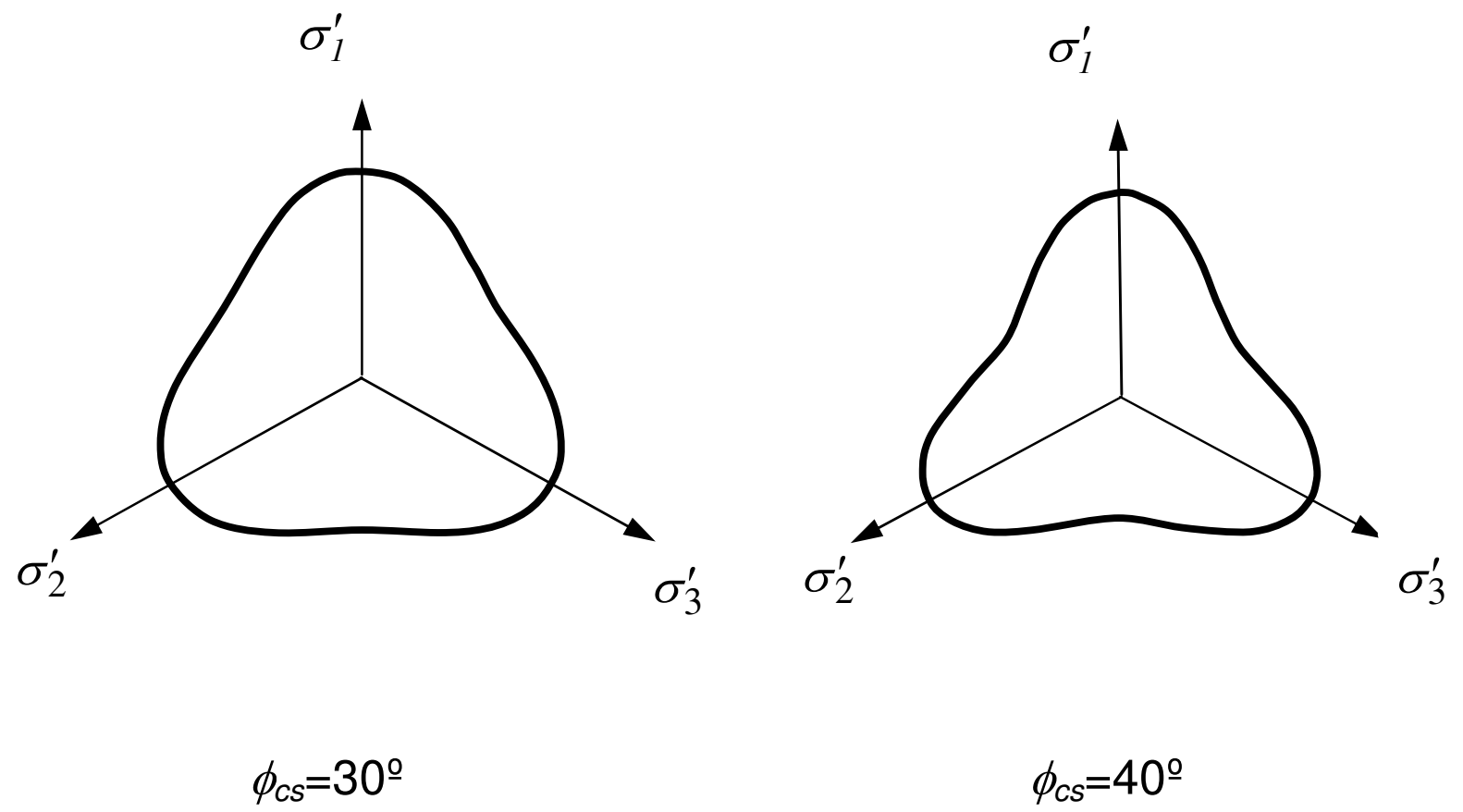

Figure 3: Failure surface in deviatoric according to equation (15)

$$
\text { for } \phi_{c s}=30^{\circ} \text { and } \phi_{c s}=40^{\circ} \text {. }
$$




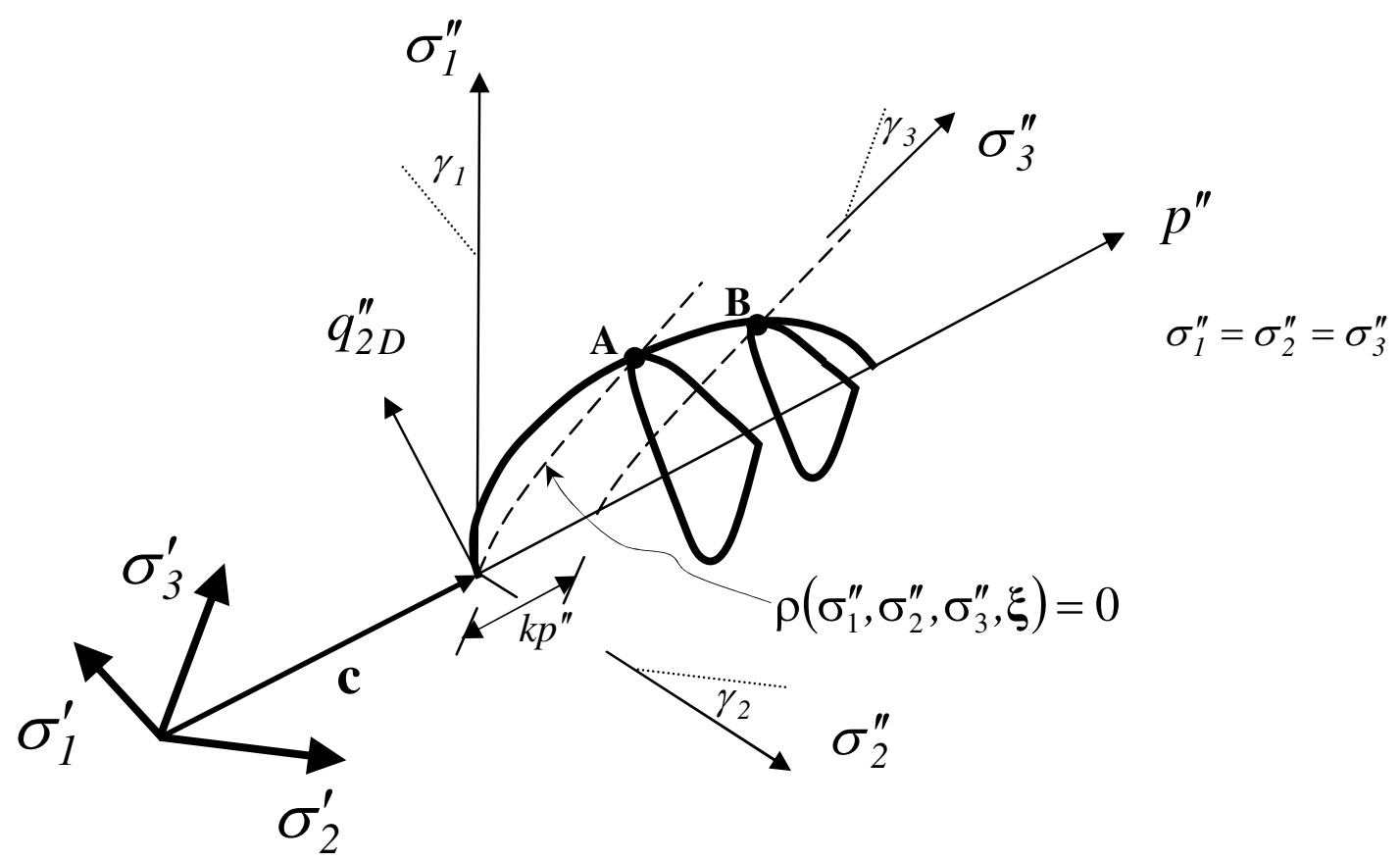

Figure 4: Schematic representation of proposed generalization technique in the general stress space with a non-linear operator. 


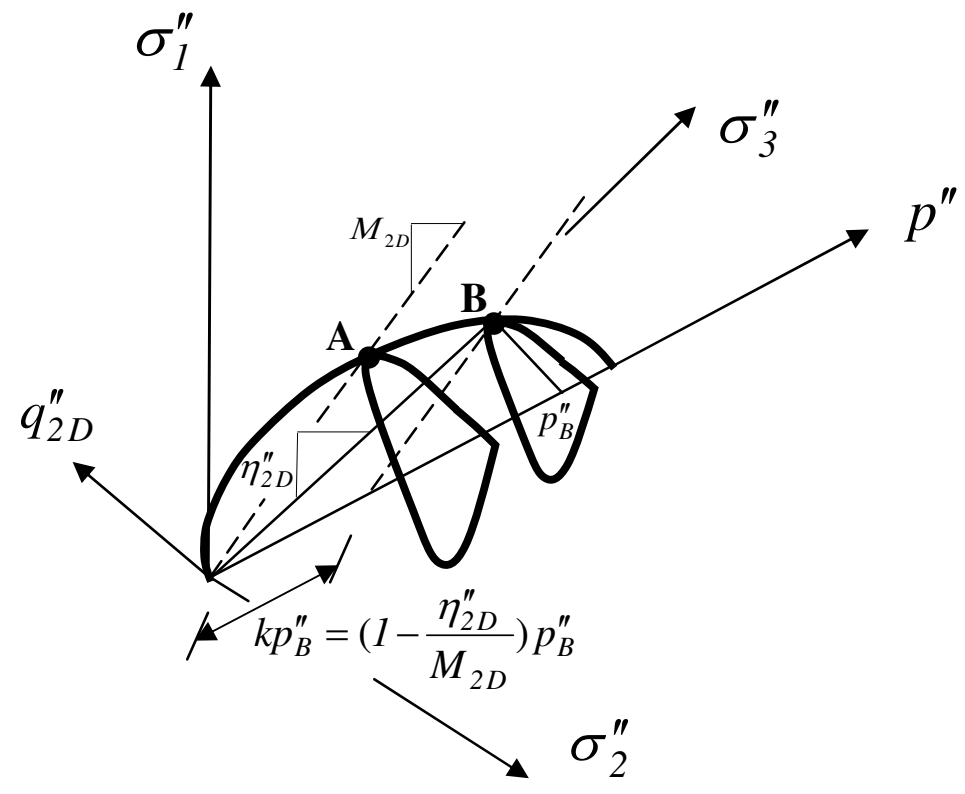

Figure 5: Schematic representation of proposed generalization technique in the local coordinate system with a linear operator. 


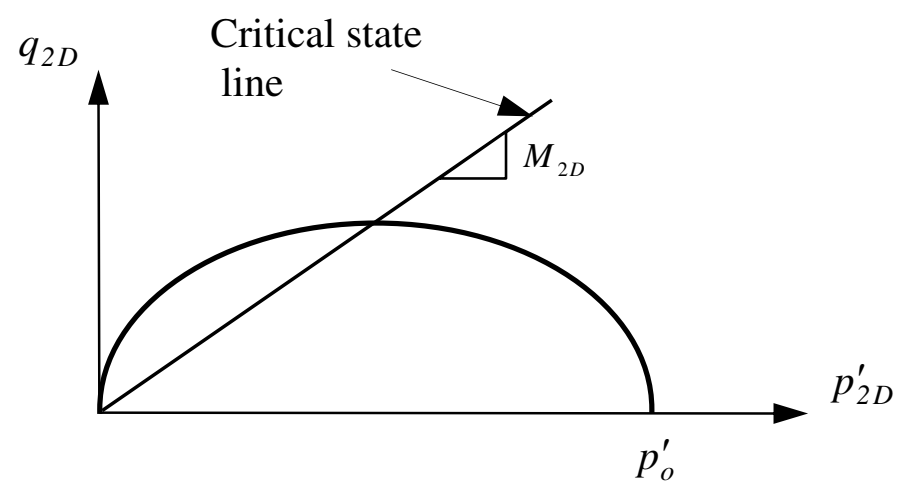

Figure 6: Modified Cam Clay model. 


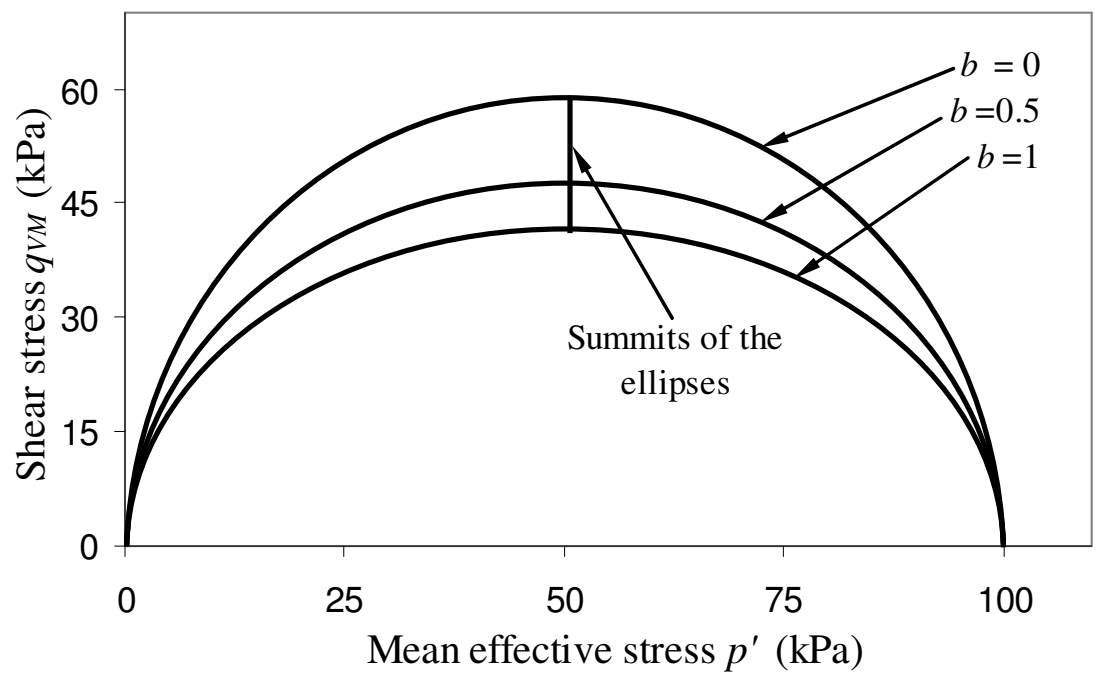

Figure 7: Intersections of the yield surface with different $b$ for the proposed model. 


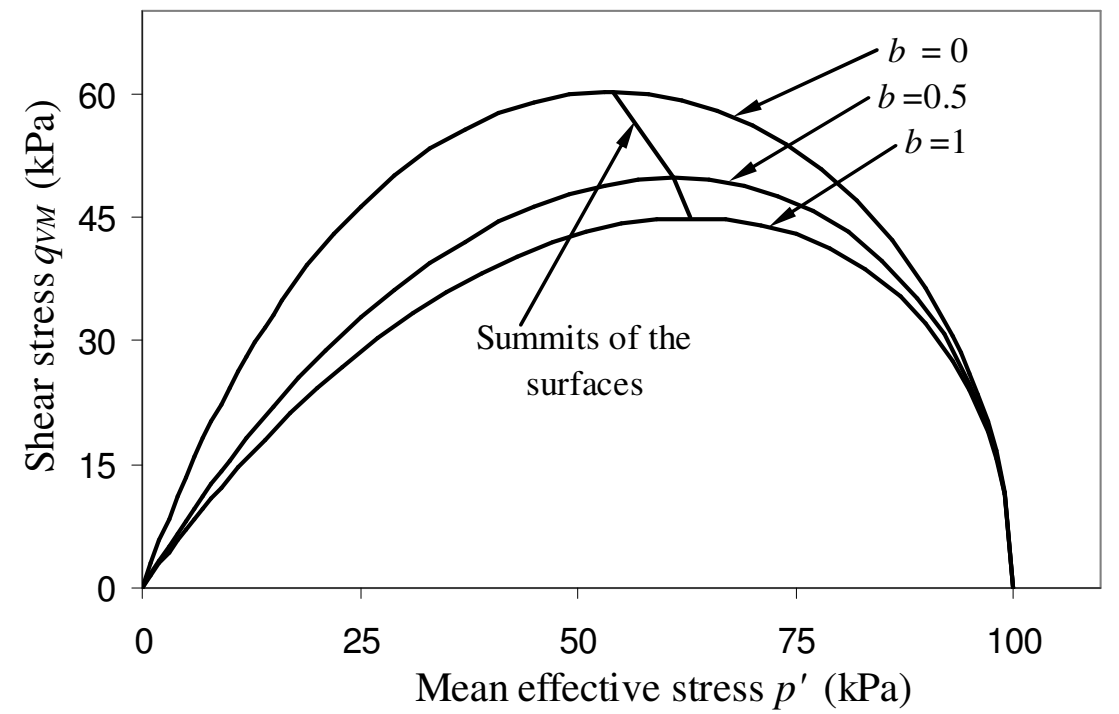

Figure 8: Intersections of the yield surface for the model extended via generalized shear stress ratio. 


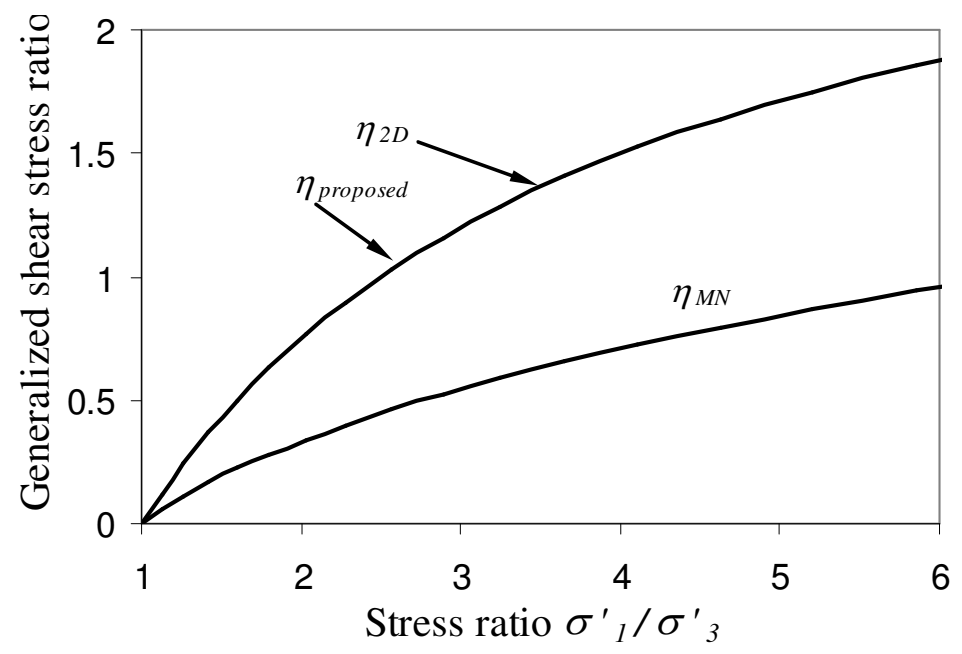

Figure 9: Generalized shear stress ratios via various methods for axysymmetical stress states. 


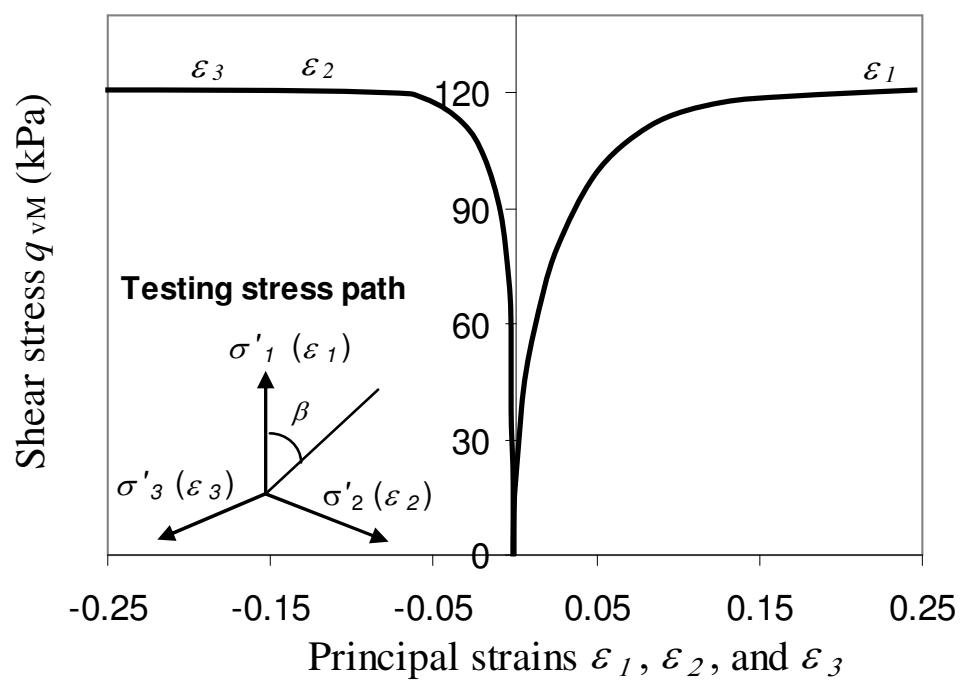

Figure 10: Principal strains simulated via the proposed method for a test with $b=0\left(\beta=0^{\circ}\right)$. 


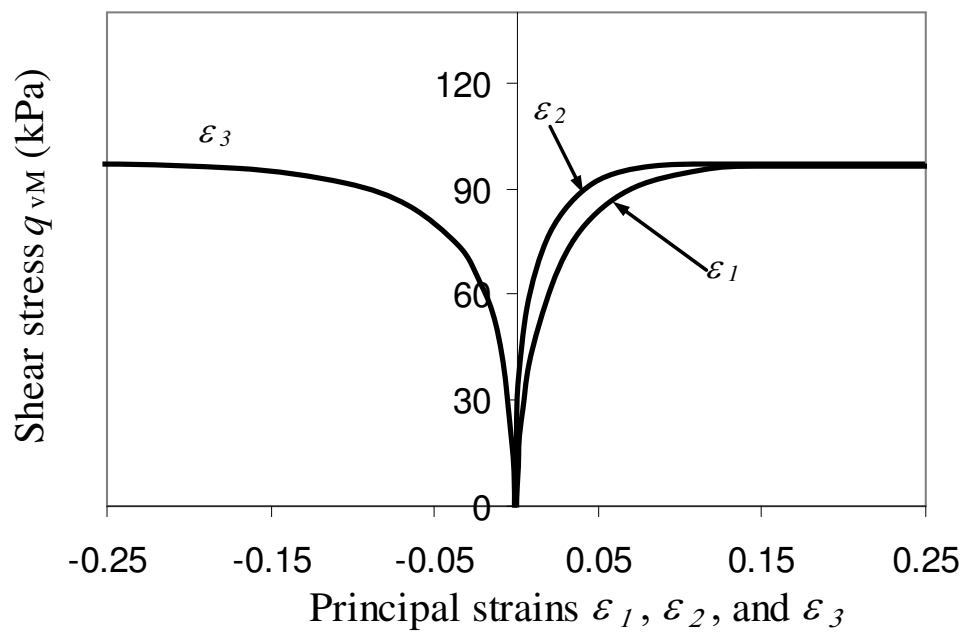

Figure 11: Principal strains simulated via the proposed method for a test with $b=0.5$

$$
\left(\beta=30^{\circ}\right) .
$$




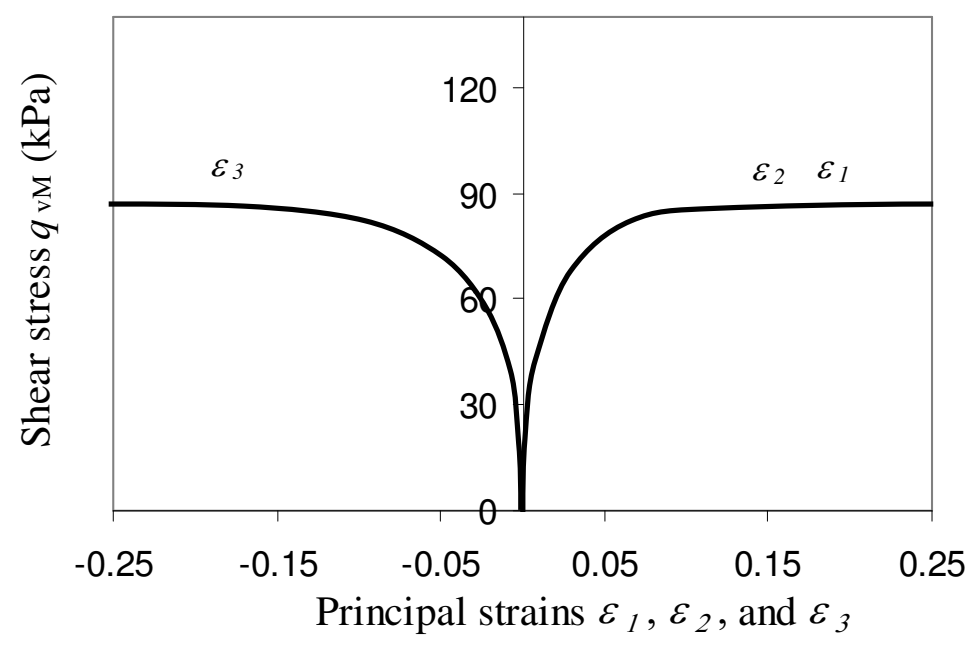

Figure 12: Principal strains simulated via the proposed method for a test with $b=1\left(\beta=60^{\circ}\right)$. 


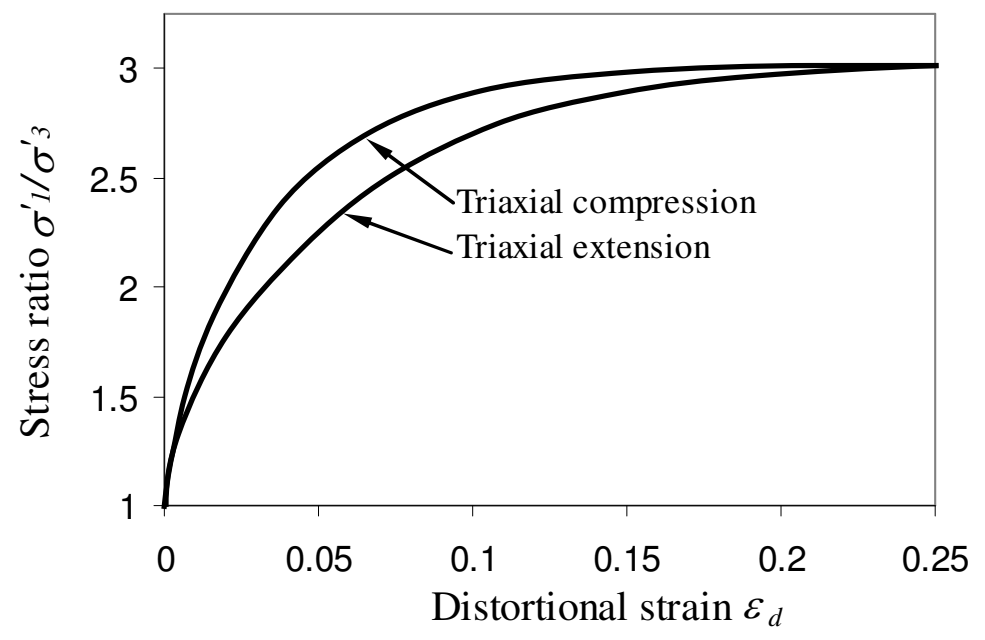

Figure 13: Shear stress ratio and distortional strain relationship simulated via the proposed method. 


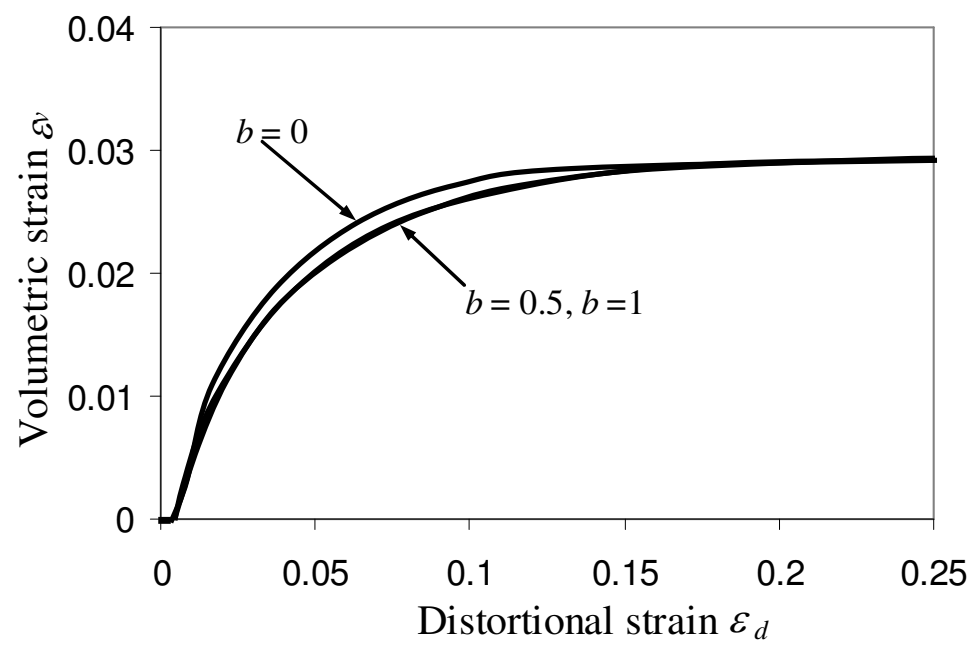

Figure 14: Volumetric and distortional strain relationship simulated via the proposed method. 


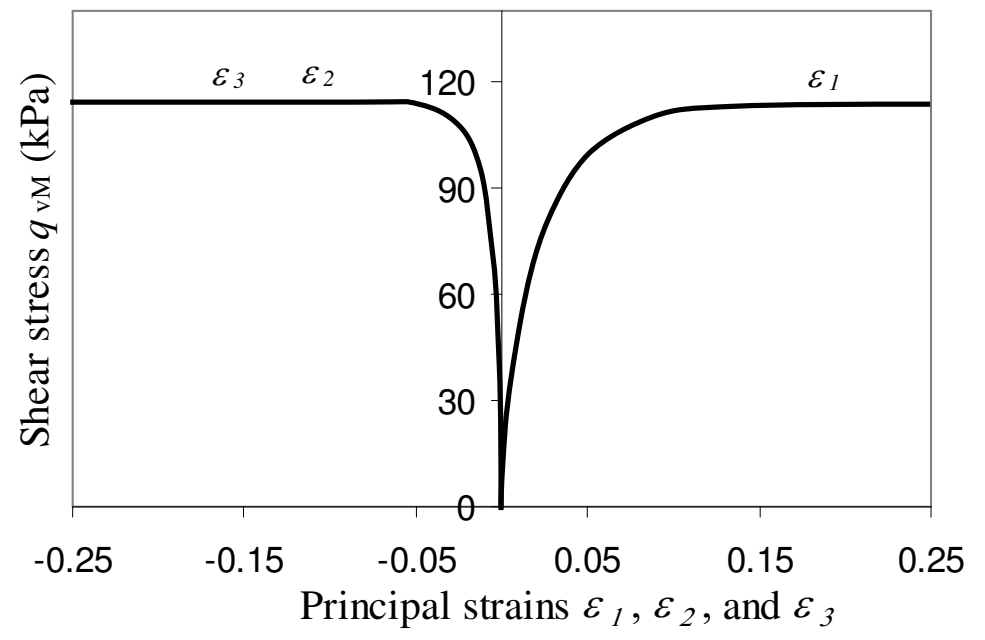

Figure 15: Principal strains simulated via the generalized stress ratio method for a test with $b=0\left(\beta=0^{\circ}\right)$. 


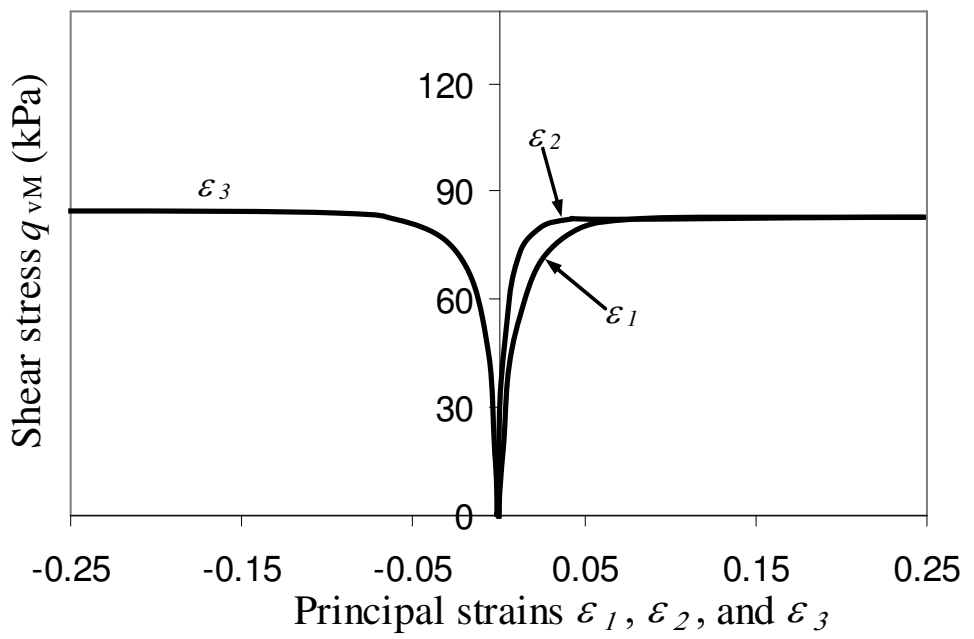

Figure 16: Principal strains simulated via the generalized stress ratio method for a test with $b=0.5\left(\beta=30^{\circ}\right)$. 


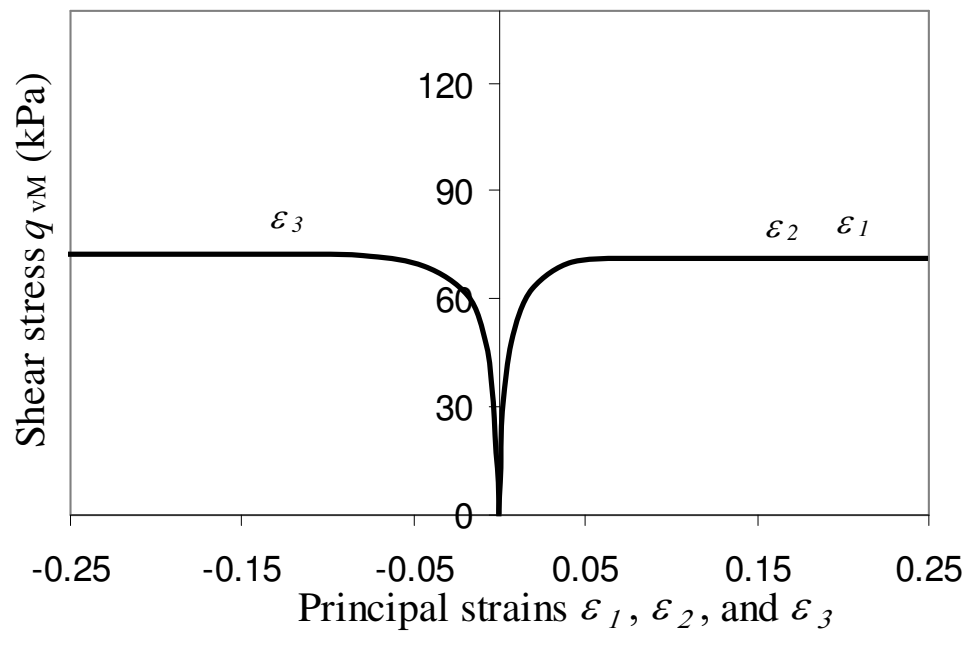

Figure 17:Principal strains simulated via the generalized stress ratio method for a test with $b=1\left(\beta=60^{\circ}\right)$. 


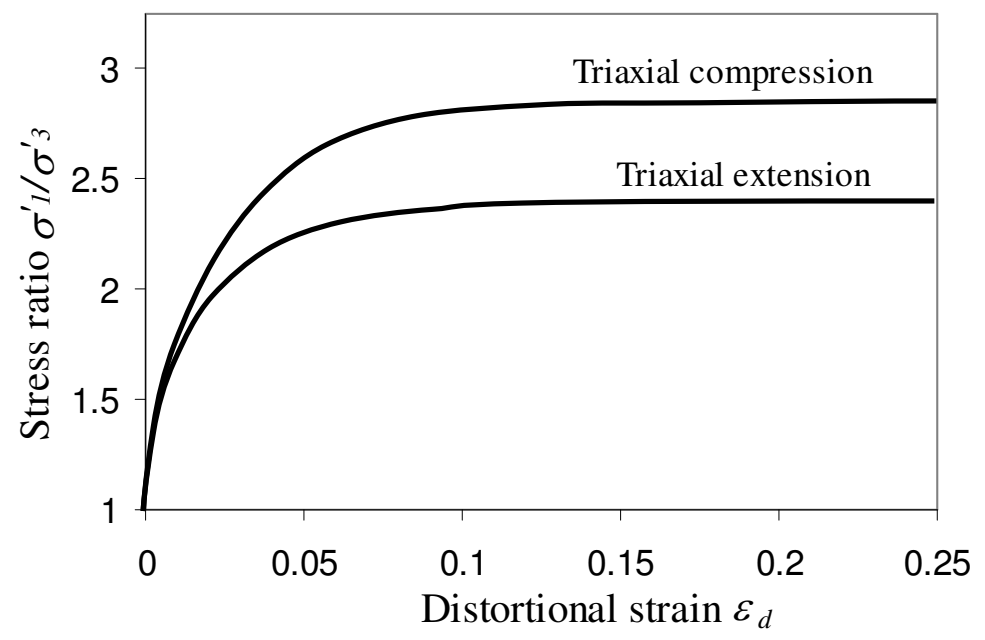

Figure 18: Shear stress ratio and distortional strain relationship simulated via the generalized shear stress ratio method. 